\title{
RAMAN SPECTRA OF PHENOTHIAZINE AND SOME PHARMACEUTICAL DERIVATIVES
}

\author{
Barbara Kure* and Michael D. Morris \\ Department of Chemistry, University of Michigan, Ann Arbor, MI 48104, U.S.A.
}

(Received 22 August 1975. Accepted 25 September 1975)

Phenothiazınes are widely used as antipsychotic drugs and for this reason there has been much effort devoted to the spectroscopic characterization of these compounds. Although the infrared spectra of phenothiazines have been well characterized, ${ }^{1}$ there have been no reports of the Raman spectra. Raman spectrometry appears useful in the identification of phenothiazines because of the presence of carbon-sulphur bonds and because the technique is well suited to the examination of solids, the physical form of most pharmaceutical preparations. In the present communication we report the spectra of several solid phenothiazines in the $600-1600-\mathrm{cm}^{-1}$ region and present preluminary assignments of the major bands.

\section{EXPERIMENTAL}

The formulae of the substituted phenothrazines studied are presented in Table 1. Compounds I (trifluoropromazine hydrochloride) and II were obtained from the Squibb Institute. Compounds III and IV were obtained from Searle Laboratories. These compounds were used without further purification. Unsubstituted phenothiazine (Aldrich Chemical Co.) was recrystallized from benzene.

Raman spectra were recorded on a Spex 1401 double monochromator equipped with a cooled RCA C31034 photomultiplier and both photon-counting and d.c. detection systems. He-Ne $632 \cdot 8-\mathrm{nm}$ excitation $(25-35 \mathrm{~mW}$ at

* Present address: Department of Chemistry, University of Wisconsin, Madison, Wis. 53706, U.S.A. the sample) was employed. Samples were irradiated for about $30 \mathrm{~mm}$ in the laser beam to remove residual fluorescence. Samples were introduced into the laser beam as powders contained in standard glass melting-point capillaries. All spectra were recorded with $10 \mathrm{~cm}^{-1}$ resolution.

\section{RESULTS AND DISCUSSION}

The Raman spectra of the phenothiazines examined are presented in Table 2. Because the samples were examined as polycrystalline solids, depolarization ratios were not taken.

The phenothiazıne spectra can be interpreted as derived from the spectra of diphenylamine, diphenyl sulphide and the various saturated compounds which make up the sidechains. Such an approach is valid, since the phenothiazine is known to be folded about the $\mathbf{S}-\mathbf{N}$ axis, with a dihedral angle between the phenylene rings of about 140-154, depending on the substitution pattern. ${ }^{2,3}$ Thus, the two phenylene rings are more or less independent and the spectra will be closely related to those of simpler substituted benzenes. The ring mode dominated by symmetric phenylene-sulphur stretching appears at $670-680 \mathrm{~cm}^{-1}$ for the phenothiazines investigated. In diphenyl sulphide (liquid) this vibration occurs at $668 \mathrm{~cm}^{-14}$ The differences may be attributed to both the difference in the phase and to the presence of an ortho-substituent in phenothiazine and possibly to the difference in the C-S-C bond angle. This band $1 \mathrm{~s}$ in the same region as the $\mathrm{C}-\mathrm{C}$ out-of-plane deformation, which is responsible for the infrared absorption

Table 1 Formulae of substituted phenothiazines

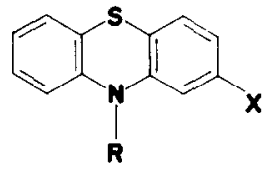

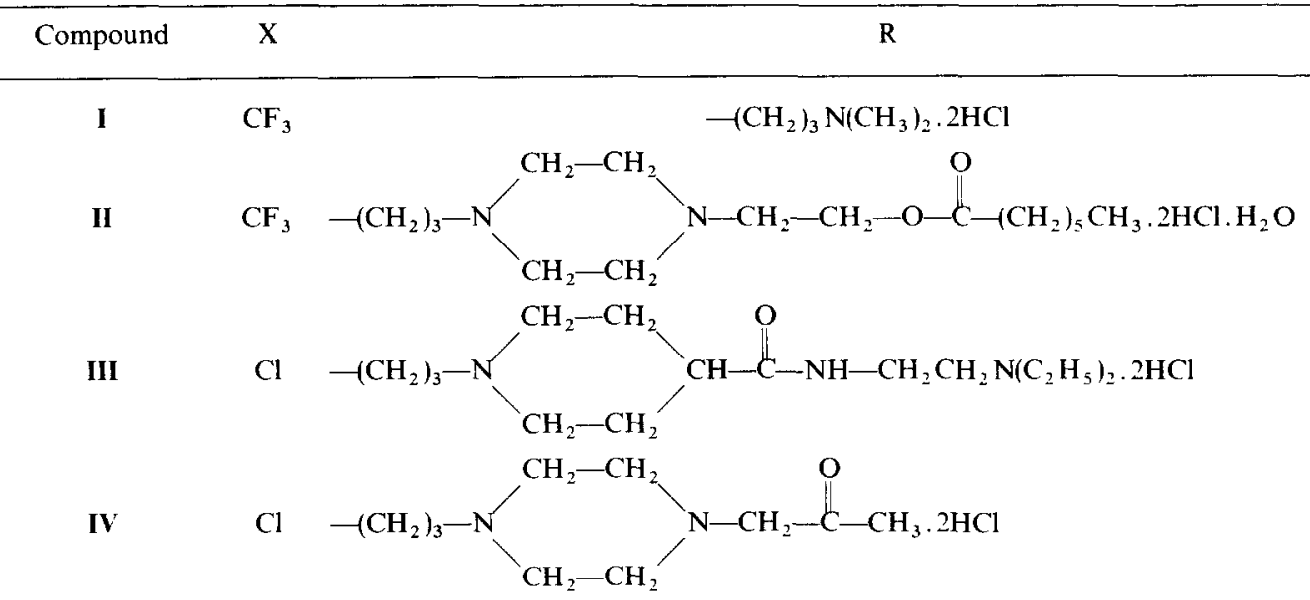


Table 2. Raman spectra of phenothiazines

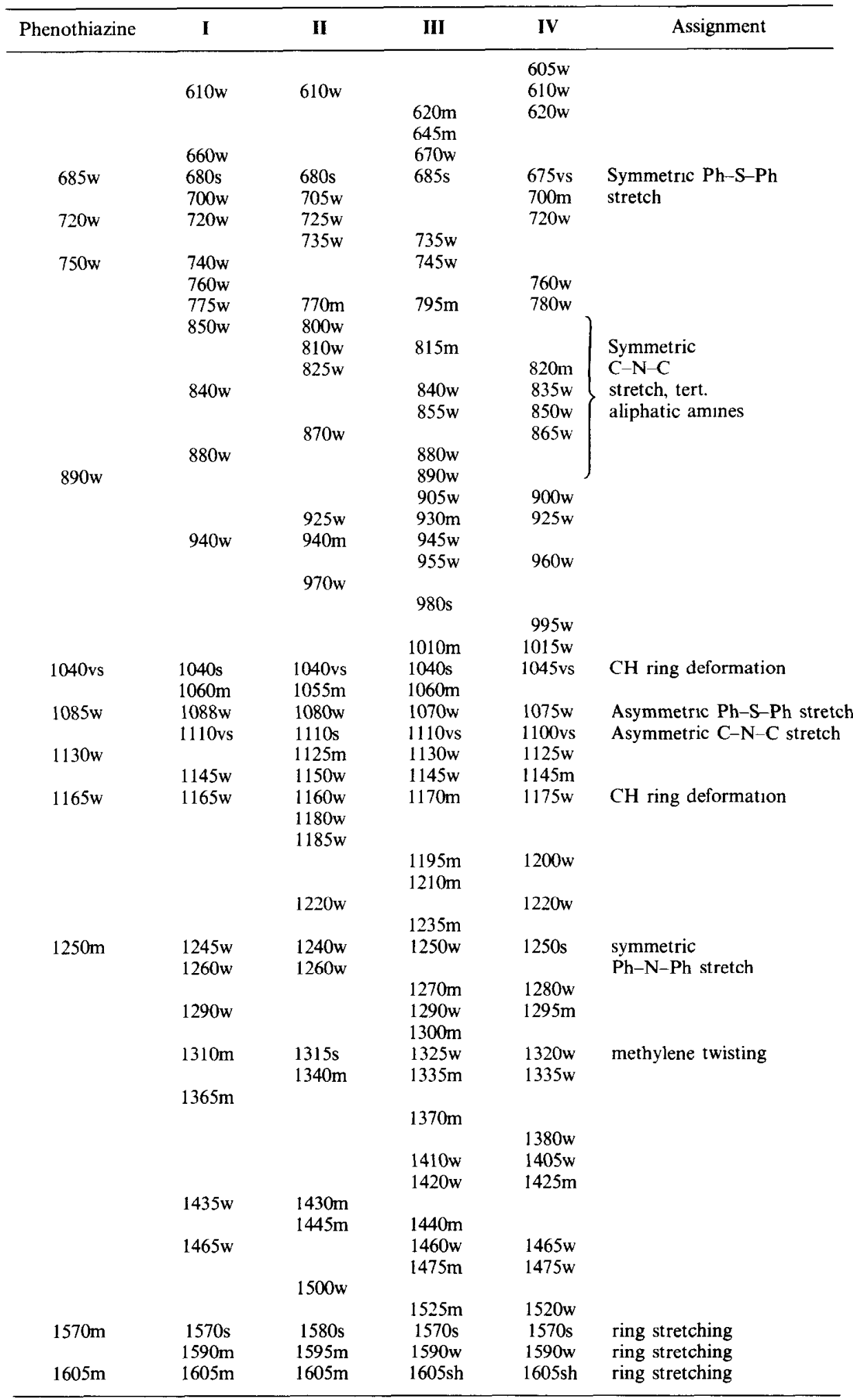


in the $690 \mathrm{~cm}^{-1}$ region. ${ }^{4.5}$ Overlap with this mode, whuch is active in ortho-disubstituted benzenes, ${ }^{6}$ cannot be ruled out.

The asymmetric phenylene-sulphur stretches occur in the $1070-1090-\mathrm{cm}^{-1}$ region. In diphenyl sulphide this band occurs at around $1080 \mathrm{~cm}^{-1,4,5}$

The medium intensity symmetric phenylene-nitrogen stretch is found at $1240-1250 \mathrm{~cm}^{-1}$. In solid diphenylamine, this vibration is observed in the infrared at $1250 \mathrm{~cm}^{-1}$.

The intense $1040 \mathrm{~cm}^{-1}$ band we assign to a $\mathrm{C}-\mathrm{H}$ ring deformation. This is a strong band at $1040 \mathrm{~cm}^{-1}$ for diphenylamine and at $1026 \mathrm{~cm}^{-1}$ for diphenyl sulphide. ${ }^{4}$ A weaker $\mathrm{C}-\mathrm{H}$ deformation occurs at $1165-1175 \mathrm{~cm}^{-1}$ and is observed at $1153 \mathrm{~cm}^{-1}$ for diphenyl sulphide.

In the spectrum of unsubstituted phenothiazine a pair of ring modes occurs at 1570 and $1605 \mathrm{~cm}^{-1}$. These are the two components of a ring-stretching mode $(1$ and $k$ in Whiffen's notation). In the spectra of the ring-substituted phenothiazines, three bands occur in this region, owing to the non-equivalence of the two phenylene rings. We have not attempted detailed assignments of the bands due to vibrations of the alkyl side chains. We present only a few general assignments, but do not attribute bands to individual ammes or alkyl groups.

The pharmaceutical derivatives have a group of bands between 800 and $900 \mathrm{~cm}^{-1}$, due to symmetric $\mathrm{C}-\mathrm{N}-\mathrm{C}$ stretches of the tertiary amino groups. ${ }^{B}$ The asymmetric C.N.C stretches appcar as strong bands at $1100-$ $1110 \mathrm{~cm}^{-1}$. Similarly, the band at $1310-1325 \mathrm{~cm}^{-1}$ is assignable to a methylene twisting mode of the alkyl chains common to all of the substituted phenothiazines. ${ }^{9}$
Further refinement of these spectra and their extensions to higher and lower frequencies are in progress and will be reported at a later date.

Acknowledgements - We wish to thank Miss B. Stearns, Squibb Institute for Medical Research and Dr. Kurt Rorig, Searle Laboratories, for samples of the compounds used in this work.

\section{REFERENCES}

1. C Bodea and I. Silberg, Advances in Heterocyclic Chemistry, Vol. 9. p. 335. Academic Press. New York, 1968.

2. J. J. H. McDowell, Acta Cryst, 1969, B25, 2175.

3. J. D. Bell, J. F, Blount, O. V. Briscoe and H C. Freeman, Chem. Comm., 1968, 1656.

4. J. H. S. Green, Spectrochim. Acta, 1968, 24A, 1627

5. B. Nagel, T. Steiger, J. Fruwert and G. Geiseler, ihid. $1975,31 \mathrm{~A}, 255$.

6. F. R. Dollish. W. G. Fateley and F. F. Bentley, Characteristic Raman Frequenctes of Organic Compounds, $\mathrm{p}$. 175. Wiley-Interscience, New York. 1974.

7 A. P. Rudko, I. N. Chernyuk, Yu. S. Rozum and G. T. Pilyugin, Ukr, Khim. Zh, 1968, 34, 1275; Engl Trans. Sov. Prog. in Chew. 1968, 34, 67.

8. F. R. Dollish. W. G. Fateley and F. F. Bentley, Characteristic Raman Frequencies of Organic ' Compounds, $\mathrm{p}$. 35. Wiley-Interscience, New York, 1974.

9. Idem, ihid., p. 1 .

Summary-The laser-Raman spectra of several phenothiazines in the region $600-1600 \mathrm{~cm}^{-1}$ are reported. The major bands are assigned to ring modes or to vibrations of the alkyl side-chains.

\title{
THE DETERMINATION OF METALLIC IRON IN THE PRESENCE OF FAYALITE
}

\author{
I. J. BEAR and P. R. STRODE \\ CSIRO Division of Mineral Chemistry, P.O. Box 124, Port Melhourne, Victoria 3207, Australia
}

(Received 20 August 1975. Accepted 7 October 1975)

A current investigation in these laboratories, into the treatment of low-grade tin ores, involved the reduction of tin calcines under various conditions. During the analysis of these reduced calcines difficulty was encountered in determining metallic iron. The reduced samples contained metallic iron, tin and tin-iron alloys, tin and iron oxides. aluminium-miron oxides (hercynite), and iron silicate (fayalite, $2 \mathrm{FeO} . \mathrm{SiO}_{2}$ ).

It was confirmed that metallic aron can be determined satusfactorily in the presence of rron oxides (both ferrous, ferric and complex oxides such as hercynite) by methods such as those of Kraft and Fischer ${ }^{1}$ or Blum and Sear ${ }^{2}$ whereby the sample is treated with bromine in methanol under reffux conditions. The oxide residue is subsequently filtered off, washed with methanol, and, following the evaporation of alcohol from the filtrate, the iron equivalent to the metallic uron content is determined in dilute hydrochloric acid solution.
The method above, however, does not give accurate results for metallic iron when iron silicates such as fayalite are present in the sample to be analysed. The errors arise because fayalite is attacked by hydrogen bromide which apparently forms in the alcoholic solution of bromine even when moisture is rigorously excluded. ${ }^{3}$ In Fig. $1 .(a)$ and $(b)$, are shown portions of the X-ray diffraction patterns of a synthetic sample containing iron (II) oxide, silica, and approximately $6 \%$ fayalite before and after treatment with bromine in methanol. It is clear that most of the fayalite has been dissolved during this treatment.

Several variations of the method of Kraft and Fischer ${ }^{1}$ were studied in order to overcome this problem. It was found that whereas iodine in methanol did not attack the fayalite, the metallics-tin. mon, and iron-tin alloys-were halogenated only slowly. A more satisfactory variation was to treat the sample with bromine alone under reflux, then evaporate all excess of bromine before extracting the iron 